\title{
Paper-like Surface Microstructure Fabricated on a Polymer Surface by Femtosecond Laser Machining
}

\author{
Kenji GoYa ${ }^{\dagger}$ and Yusuke FuchIwaKI \\ Health Research Institute, National Institute of Advanced Industrial Science and Technology, 2217-14 Hayashi, \\ Takamatsu, Kagawa 761-0395, Japan
}

\begin{abstract}
In this study, we demonstrate the precise control of fluid flow using femtosecond (FS) laser-induced microstructures. A microgroove structure inscribed on a poly(methyl methacrylate) (PMMA) substrate functions as a superhydrophilic membrane similar to paper. We first estimated the flow rate for pure water on microgrooves fabricated at various laser fluences in the range from 9.2 to $100.8 \mathrm{~J} / \mathrm{cm}^{2}$. The results showed that the flow rate could be tuned in the range from 0.30 to $12.07 \mu \mathrm{L} / \mathrm{s}$ by varying the laser irradiation parameters. The fluid flow was reproducible, with a calculated relative standard deviation (RSD\%) of less than $8 \%$ in the flow rate. We then fabricated a microfilter for blood separation and estimated its filtration ability using artificial blood containing resin microparticles. This method would be useful in a technology related to a paper-based diagnostic device for precise reagent manipulation.
\end{abstract}

Keywords Paper-like microstructure, flow control, flow rate, laser machining

(Received August 28, 2017; Accepted October 19, 2017; Published January 10, 2018)

\section{Introduction}

Paper is a well-known material and is used in a wide range of fields because of its inherent abilities to absorb and transfer liquids through capillary force driven by porous structures and without external equipment. Since paper was introduced as a fluidic control device ${ }^{1}$ for biochemical assays, paper-based fluidic devices ${ }^{2-11}$ have been developed as a new diagnostic platform and fluid flow control technique because such systems require no external power or off-chip components and include no moving parts.

The flow channel of paper-based microfluidic devices consists of a hydrophilic area and hydrophobic wax walls to control the spreading of fluid. The flow rate can also be controlled by varying parameters such as permeability, channel width, channel length, and channel height, where the flow characteristics are described by Darcy's law. ${ }^{12}$ The flow rate of a fluid through the paper channel is proportional to the channel's cross-sectional area, length, and permeability and to the fluid's viscosity. Several researchers have attempted to change the permeability ${ }^{8,13-16}$ of paper to control the fluid flow. In particular, Chu et al. ${ }^{8}$ and Lutz et al. ${ }^{14}$ demonstrated lateral flow control by changing the permeability via infiltration of sugars into paper strips. The applicability of these methods to paper channels is limited in terms of device size and sample consumption because of the devices' manufacturing procedure. Furthermore, previous methods have required an additional process in which a certain

\footnotetext{
† To whom correspondence should be addressed.

E-mail: gouya-k@ile.osaka-u.ac.jp

K. G. present address: Institute of Laser Engineering, Osaka University, Room 330, Bldg. I, 2-6 Yamadaoka, Suita, Osaka 565-0871, Japan.
}

amount of hydrophobic wax or sugar solution is added after the paper channel has been fabricated.

Paper-like microstructures designed and fabricated on a polymer surface could be used instead of porous paper structures for biological assay systems without external components. We have recently demonstrated antibody immobilization on a hemiwicking ${ }^{17}$ polymer surface prepared using femtosecond (FS) laser microstructuring. ${ }^{18}$ FS laser microstructuring enables the creation of superhydrophilic microgrooved structures that provide paper-like features such as liquid migration and absorptivity driven by capillary force. Using such hydrophilic structures, an antibody can selectively and precisely be immobilized in a spatially localized area. Precise machining using an FS laser-referred to as nonthermal ablation ${ }^{19-21}$ - has received much attention because the nonthermal effect allows particular regions of a substrate to be modified without thermally induced collateral damage; functional structures can thereby be flexibly provided for controlling microliter-volume liquids. ${ }^{22,23}$

In this paper, we demonstrate using FS laser-induced microgrooved structures inscribed on a poly(methyl methacrylate) (PMMA) substrate to precisely control fluid flow without any external components. First, we estimated the flow rate for pure water dispensed on microgrooves fabricated using various laser parameters and with flow channels of various sizes. We then designed a microfilter to estimate the filtration ability using artificial blood containing resin microparticles as red blood cells.

\section{Experimental}

\section{Laser fabrication system}

Figures 1(a) and 1(b) show schematics of the experimental setup and method, respectively, used to fabricate microgrooves 
(a)
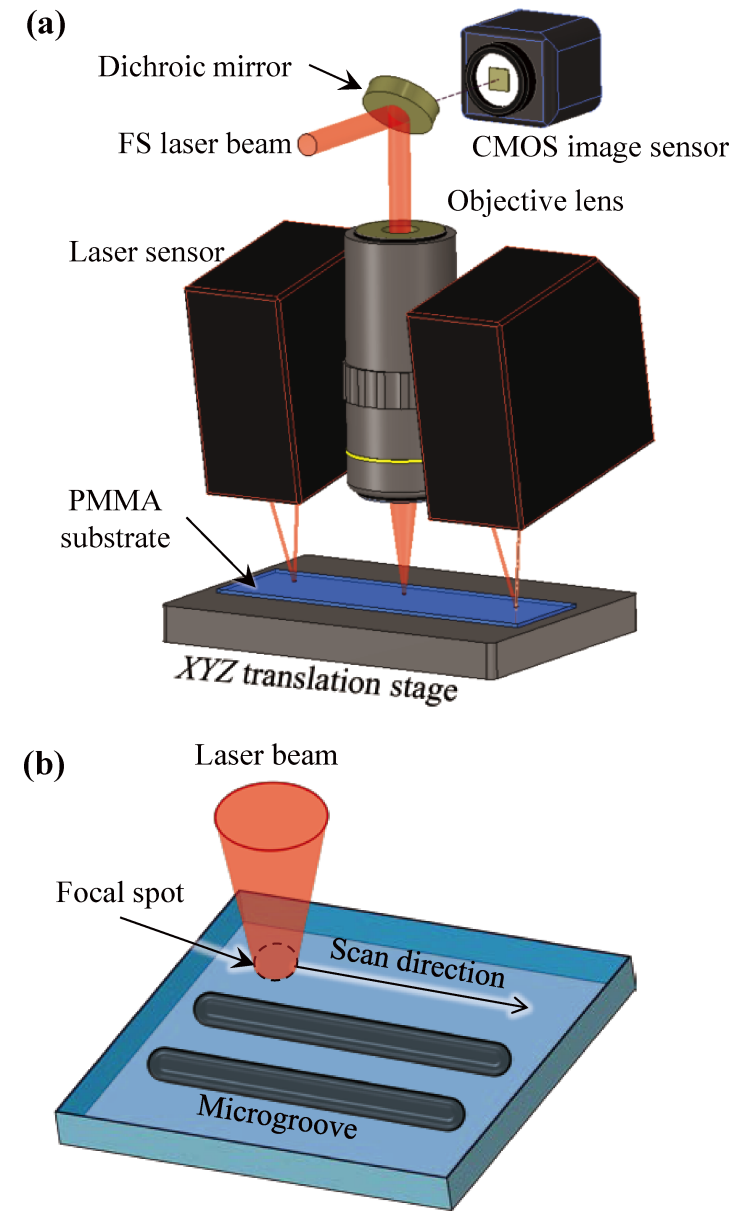

Fig. 1 FS laser system used to inscribe microgroove structures onto a PMMA substrate. on PMMA substrates. A PMMA substrate was mounted on a three-dimensional translation stage, and the position of the laser focal point was adjusted by moving the stage in the $X, Y$, and $Z$ directions. As evident from Fig. 1(a), laser displacement sensors (LKG-35A, Keyence) were installed on both sides of the objective lens to precisely adjust the laser focal point and to reduce the unfavorable tilt of the $X Y$ plane because fluctuations in the position of the laser focal point strongly influences the surface wettability of microgrooves. ${ }^{24}$ The FS laser beam (158 fs, $\lambda=780 \mathrm{~nm}$ ) with a beam diameter of $3.0 \mathrm{~mm}$ was focused onto the substrate surface by an objective lens $(\mathrm{NA}=0.28)$ after reflection by a dichroic mirror mounted to monitor the vicinity of the laser focal point with a complementary metal oxide semiconductor image sensor (CMOS; Moticam 1000, Shimadzu Rika) in the same direction as the FS laser beam. The pulse energy of the laser beam was adjusted by neutral density filter. The spot size $W$ at the focal point was calculated to be approximately $11 \mu \mathrm{m}$ on the basis of the Gaussian optics expression $W=4 M^{2} \lambda f / \pi D_{1 / e}$, where $M^{2}=1.45$ is the beam quality, focal length $f=20 \mathrm{~mm}$ (objective lens $20 \times$ ) and the laser beam diameter $D_{1 / \mathrm{e}}=2.6 \mathrm{~mm}$. The focal point was set at $0.1 \mathrm{~mm}$ in depth from the PMMA surface of the inlet, and then the beam diameter is approximately $24 \mu \mathrm{m}$ at the surface.

A laser-induced microgroove was optically inscribed on the substrate by moving the translation stage in the scan direction (Fig. 1(b)) at a scan speed of $400 \mu \mathrm{m} / \mathrm{s}$. Each microgroove was spaced at $40 \mu \mathrm{m}$ intervals and each microgroove was $6 \mathrm{~mm}$ in length. The incremental motion was controlled through a computer. Further details regarding the fabrication conditions are available in our previous work. ${ }^{18}$

\section{FS laser-induced superhydrophilic surface}

Figure 2 depicts the contact angle of a water droplet with a volume of $0.5 \mu \mathrm{L}$ on a raw PMMA $(\mathrm{a}, \mathrm{b})$ surface and on a superhydrophilic grooved structure (c, d) fabricated on a PMMA substrate. The contact angle of the water on the raw PMMA is $70.4^{\circ}$. After laser irradiation, a water droplet deposited onto the

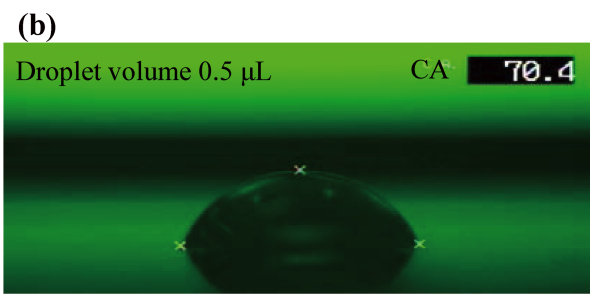

(c) PMMA with laser microgrooves

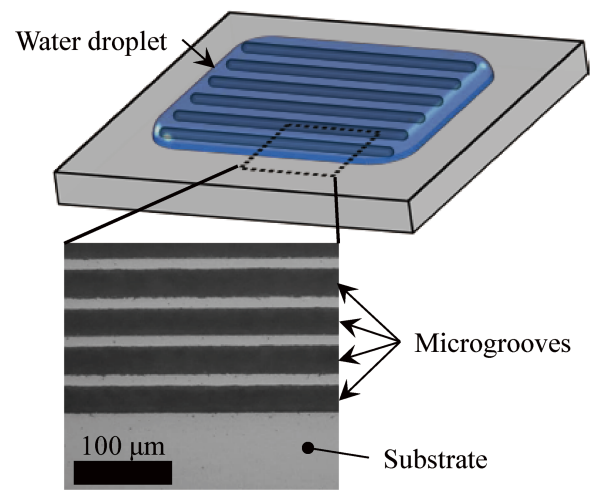

(d)

Laser ablation

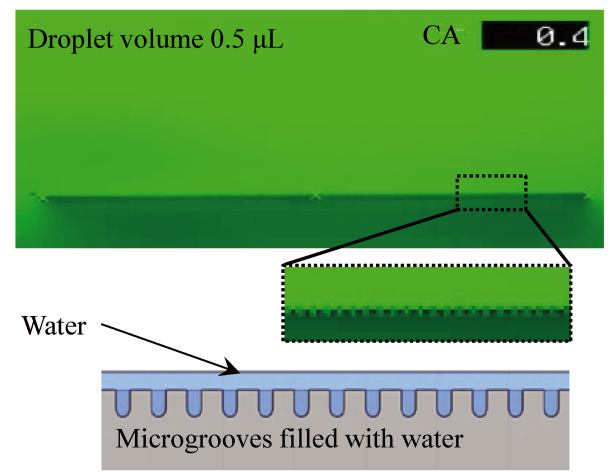

Fig. 2 Schematic and micrograph of the contact angle of a water drop on (a, b) a raw PMMA substrate and $(c, d)$ a microgrooved structure. 


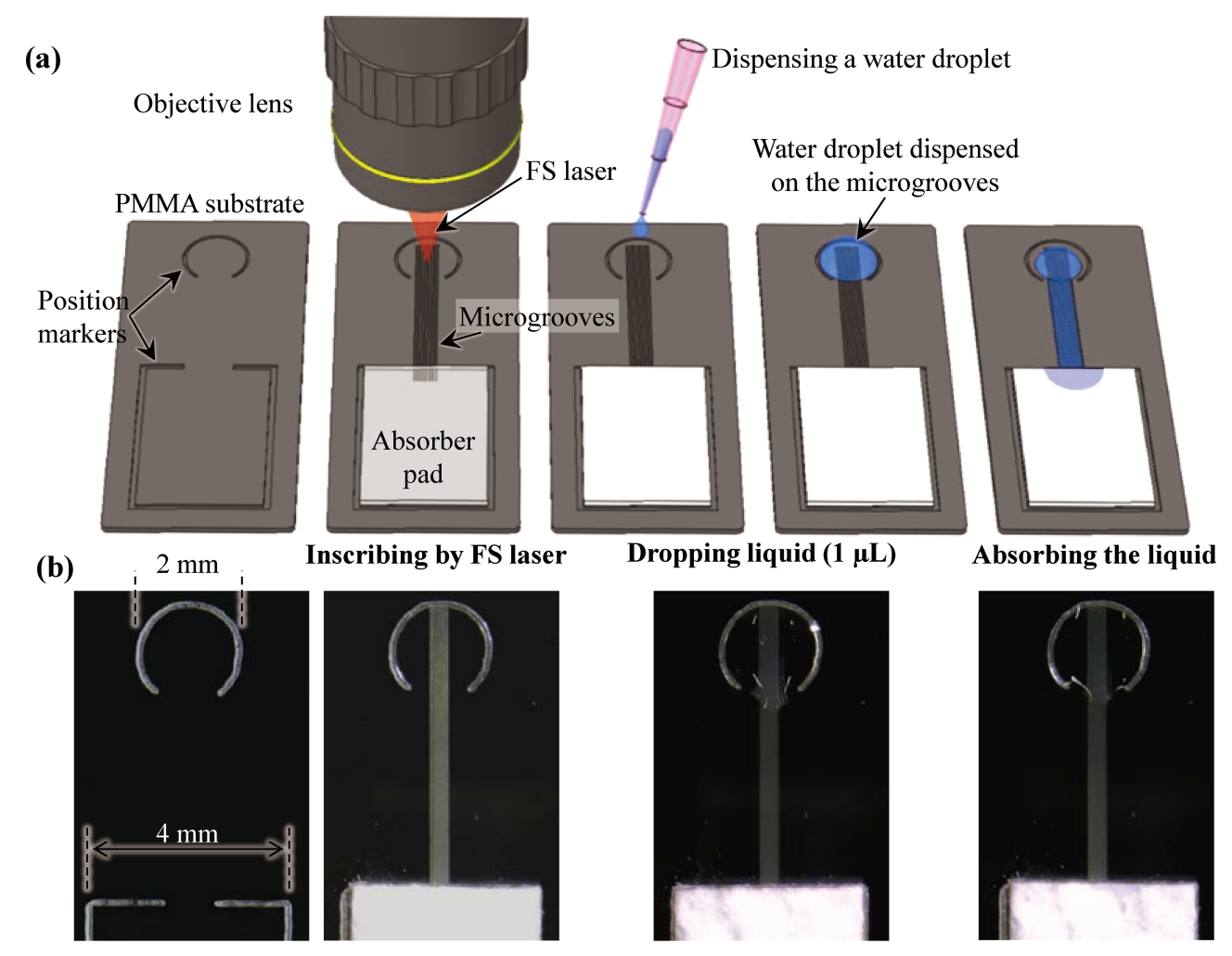

Fig. 3 Experimental setup and method to estimate the flow rate.

ablated area spread into the microgrooved texture, with a contact angle of less than $0.5^{\circ}$ (Fig. 2(d); obtained using a CA-X contact angle meter, Kyowa Interface Science). A deposited droplet immediately spread into the microgrooves because the water was driven by capillary force, resulting in a thin film shape. Such superhydrophilicity can be obtained not only by the capillary force but also by increasing the hydrophilic functional groups at the laser-irradiated area during FS laser ablation. Other investigators have experimentally demonstrated that the increase of the functional groups under FS laser ablation is proportional to the laser fluence $\left(\mathrm{J} / \mathrm{cm}^{2}\right){ }^{24,25}$ Hanada et al. anticipated that porous structures induced on the ablated area would enhance liquid wettability. ${ }^{26}$ Closer inspection of the microgrooves filled with water reveals that the water droplet was sucked into the microgrooves and stretched along the surface, where the wetting state is called hemiwicking. ${ }^{17}$ Such a microgrooved texture could be utilized as a paper-like interface between a liquid and a solid for controlling liquid migration without any external components.

\section{Flow rate measurements}

The flow rate was estimated on the basis of the time required for an absorber pad to absorb a 1- $\mu \mathrm{L}$ water droplet dispensed onto the fabricated microgrooves. Figure 3 shows schematics and micrographs of one of the microgrooved channels. The PMMA target substrate was prepared by first inscribing the position markers with a $\mathrm{CO}_{2}$ laser (Speedy400, Trotec) to determine the locations of water injection and the absorber pad. After microgrooves were fabricated through FS laser machining, an absorber pad was then placed at the outlet site (rectangular marker) for overlapping the fabricated microgrooves. A water droplet was dispensed onto the microgrooves by a precision pipette in the inlet site (circular marker). The time required for absorption of the water droplet was measured by monitoring fluid flow using a digital microscope (Dino Light Pro, Opto Science).

\section{Results and Discussion}

\section{Flow rate measurement}

To investigate the flow rate $Q$ on the microgrooved channel, we varied the grooved structure in terms of the number of microgrooves $N$ and their cross-sectional area $S$. A microgrooved channel with $N$ microgrooves $(1 \leq N \leq 20)$ is shown in Fig. 4(a). The shape of a droplet deposited in the inlet gradually changes with $N$, especially in the case of $N=20$. This behavior is attributed to the fractional ratio between the superhydrophilic grooved area and the circular inlet becoming greater than in the other cases of $N$. Note that the distance $l$ between the liquid front marked with an arrow and the outlet decreases with increasing $N$ (Fig. 4(b)). The data points and error bars correspond to the average and maximum (or minimum) values calculated from 10 replicate measurements, respectively. The measurements were carried out using 10 samples with microgrooved channels. Flow rate $Q$ is polynomially proportional to $N$. Such a polynomial increase could be caused by the change of distance $l$. The reproducibility for each sample is expressed as the calculated relative standard deviation (RSD\%) of $Q$. The flow rates for each $N(1,5,10,15,20)$ are $0.53 \pm 0.04,2.21 \pm 0.16,4.57 \pm 0.16,8.05 \pm 0.43$, and $12.07 \pm$ $0.56 \mu \mathrm{L} / \mathrm{s}$, corresponding to RSD\% values of $7.96,7.09,3.46$, 5.39 , and 4.65 , respectively. The reproducibility is similar to those obtained in a previous study using a sugar barrier. ${ }^{8}$

The surface topography of the microgrooved channel $(N=10)$ was examined by field-emission scanning electron microscopy (FE-SEM; JSM-6700FZ, JEOL) and digital microscopy (VHX5000, Keyence). Figures 4(c) and 4(d) show SEM images and a 
(a)

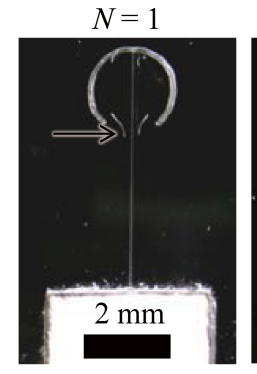

(b)

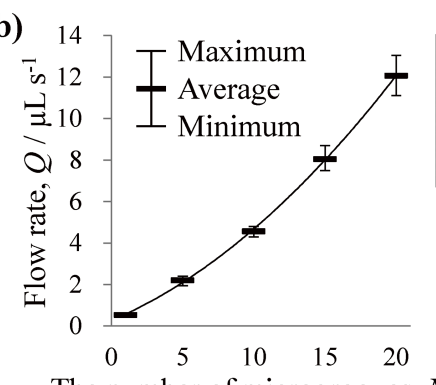

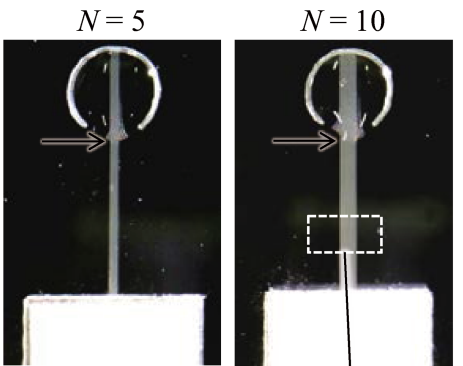

(c)

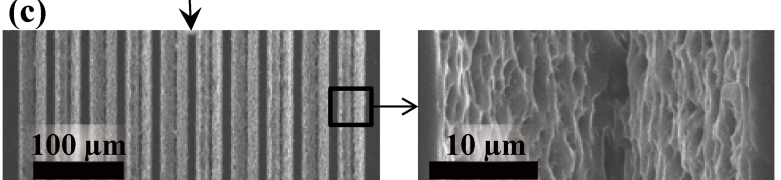

(d)

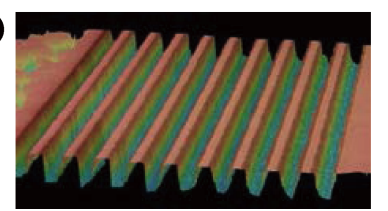

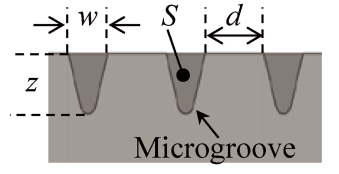

(Cross-sectional view)

Fig. 4 (a) Micrographs of microgrooved channels with $1 \leq N \leq 20$ microgrooves. The liquid front of dropped water is marked with an arrow. (b) Flow rate as a function of $N$ with the best-fit curve. The average value was obtained from 10 samples. (c) SEM images at low magnification (left) and high magnification (right). (d) 3D profile of the grooved structure $(N=10)$.

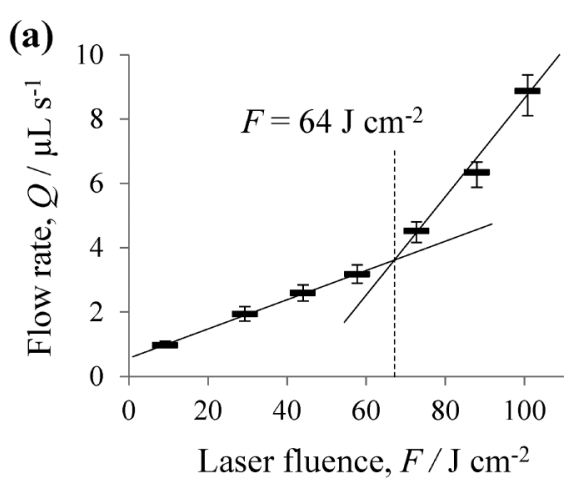

(b)

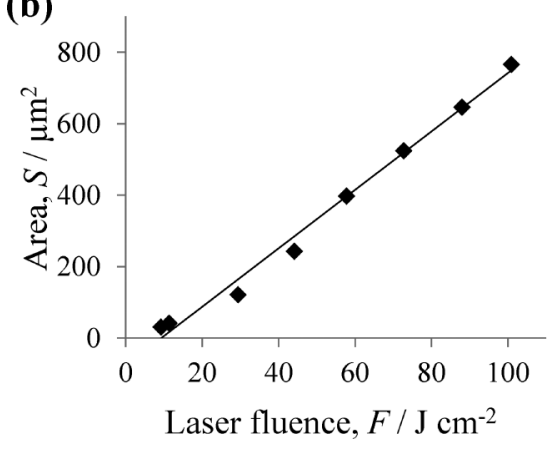

Fig. 5 The variation of flow rate $Q$ with increasing laser fluence $F$. (b) The cross-sectional area of microgrooves with respect to laser fluence with a fitting straight line.

3D profile of the grooved surface $(N=10)$. As evident from the high-magnification SEM image, we confirmed that a granular surface is generated over the inner surface of a microgroove. Such a surface pattern has generally been observed in most dielectric materials, where the scale and shape depend on laser parameters and material characteristics. ${ }^{27-29}$ The groove dimensions ( $w$, width; $d$, distance; $z$, depth) were measured from the SEM images and the 3D profile. The average values from 10 microgrooves of $w$ and $z$ were approximately 29 and $44 \mu \mathrm{m}$, respectively, where the width $w$ is defined as the width at the top of a microgroove and the depth $z$ is defined as the height between the top and the deepest bottom of a microgroove. The cross-sectional area $S$ was calculated by assuming a microgroove to be perfectly triangular.

To further investigate the characteristics of flow rate, we varied the laser fluence in the range from 9.2 to $100.8 \mathrm{~J} / \mathrm{cm}^{2}$. The results are shown in Fig. 5 and are summarized in Table 1. Figure 5(a) shows the flow rate $Q$ as a function of laser fluence $F$, where the data points and error bars correspond to the average and maximum (or minimum) values, respectively, calculated from 10 replicate measurements. The flow rate increases with increasing $F$, as does the error. Figure 5(b) shows the crosssectional area $S$ versus laser fluence $F$. Despite the crosssectional area $S$ monotonically increasing with increasing $F$, the flow rate suddenly increased when the laser fluence exceeded $64 \mathrm{~J} / \mathrm{cm}^{2}$. Such a slope change can be explained by the difference in fluid flow that occurs because of the change in the distance $d$ between microgrooves. Figsures 6(a) and 6(b) show the fluid flow of water on the microgrooves fabricated using laser fluences of 9.2 and $72.7 \mathrm{~J} / \mathrm{cm}^{2}$, respectively. The microgrooves are marked with arrows. In the case of (a) $F=9.2 \mathrm{~J} / \mathrm{cm}^{2}$, the fluid liquid passes only through the microgrooves; by contrast, the fluid flow on the surface between microgrooves is observed in the case of (b) $F=72.7 \mathrm{~J} / \mathrm{cm}^{2}$. The surface between microgrooves appears to become hydrophilic, as evidenced by laser-induced microdebris ${ }^{30,31}$ being redeposited onto the surface during laser ablation. The effect of fluid flow on the surface will be substantial when $F$ is greater than $72.2 \mathrm{~J} / \mathrm{cm}^{2}$ or when the distance $d$ between microgrooves decreases. On the basis of the results obtained in these experiments, we confirm that the flow rate can be precisely controlled by varying the laser parameters and the number of microgrooves. 
Table 1 Summary of flow rate and the dimensions of microgrooves with respect to laser fluence

\begin{tabular}{lcccccccccc}
\hline \multicolumn{1}{c}{ Definition } & Symbol & \multicolumn{1}{c}{ Unit } & \multicolumn{1}{c}{ Value } \\
\hline Laser fluence & $F$ & $\mathrm{~J} \mathrm{~cm}^{-2}$ & 9.2 & 11.4 & 29.4 & 44 & 57.8 & 72.7 & 88 & 100.8 \\
Flow rate & $Q$ & $\mu \mathrm{L} \mathrm{s}^{-1}$ & 0.3 & 1.17 & 1.96 & 2.63 & 3.18 & 4.55 & 6.35 & 9.1 \\
Width & $w$ & $\mu \mathrm{m}$ & 9.1 & 10 & 20.1 & 21.5 & 26.8 & 27.2 & 29.2 & 31.3 \\
Distance & $d$ & $\mu \mathrm{m}$ & 30.9 & 30 & 19.9 & 18.5 & 13.2 & 12.8 & 10.8 & 8.7 \\
Depth & $z$ & $\mu \mathrm{m}$ & 7.0 & 8.4 & 12.1 & 22.6 & 29.7 & 38.6 & 44.3 & 49.0 \\
Cross-sectional area & $S$ & $\mu \mathrm{m}^{2}$ & 32 & 42 & 122 & 243 & 398 & 525 & 647 & 767 \\
\hline
\end{tabular}

(a) $F=9.2 \mathrm{~J} \mathrm{~cm}^{-2}$

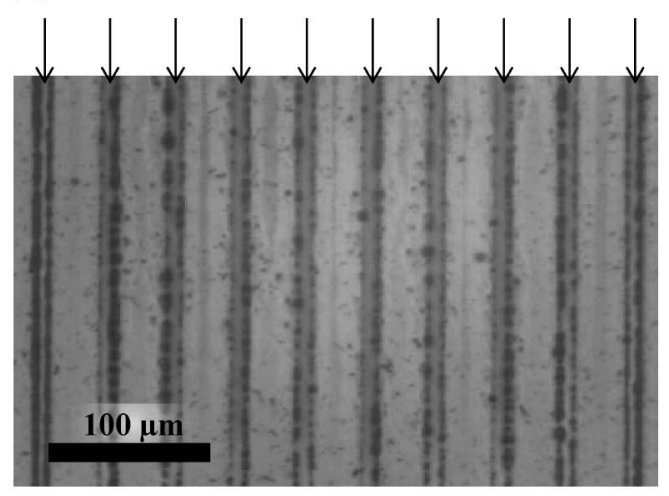

(b) $F=72.7 \mathrm{~J} \mathrm{~cm}^{-2}$

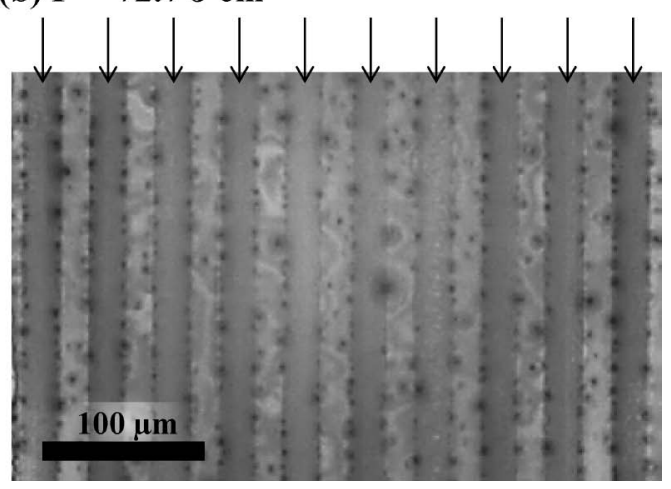

Fig. 6 Micrograph of microgrooves during liquid transfer. Each microgroove is marked with an arrow.

\section{Artificial blood filtration}

To demonstrate the filtration ability of the designed microgrooves, we evaluated their filtration performance using artificial blood (PB-10, Yamashina Seiki). The artificial blood was synthesized to be the same as human blood in hematocrit and viscosity; it was adjusted to a hematocrit of $45 \%$ and a viscosity of $2.0 \mathrm{cP}$ at $25^{\circ} \mathrm{C}$. The artificial blood cells were made from resin particles with a mean diameter $R=10 \mu \mathrm{m}$.

We prepared microgrooved channels to compare the filtration ability in the case of groove widths (a) $w=20.1 \mu \mathrm{m}$ and (b) $w$ $=9.1 \mu \mathrm{m}$, corresponding to laser fluences of 29.4 and $9.2 \mathrm{~J} / \mathrm{cm}^{2}$, respectively, as shown in Fig. 7. When the width $w$ was set to $20.1 \mu \mathrm{m}$ (Fig. 7(a): $R<w$ ), the particles flow into microgrooves and reach the end of the channel. Even though most of the particles remain in the inlet area, the filtration performance is poor. By contrast, for the case of $w=9.1 \mu \mathrm{m}$ (Fig. 7(b): $R>w$ ), the particles with $R=10 \mu \mathrm{m}$ are dammed by the microgrooves and remain entirely in the inlet. However, the smaller particles $(<4 \mu \mathrm{m})$ were found at the end of the microgrooves, as marked with a red circle in Fig. 7(b). The results indicate that the resin particles can be filtered and that the filtration ability is approximately controlled through adjusting the microgroove width or depth. Artificial plasma could be selectively separated from resin particles with microgrooved structures through careful adjustment of the microgrooves. In addition, the effect of the granulated inner surface (Fig. 4(c)) should be considered in future designs to enhance the filtration ability because the effect of surface tension and geometric structure strongly influences flow characteristics.

\section{Conclusions}

In this work, we demonstrated fluid flow control with a superhydrophilic microgrooved structure fabricated by FS laser direct machining. We confirmed that the laser-induced microgrooves inscribed on a PMMA surface function as a paper-like membrane with fluid-transfer and filtration capabilities. The fabricated microgrooved channel can stably transfer microliter volumes of liquids, and its reproducibility is comparable to that of methods involving wax barriers. Such stability could facilitate a quantitative and facile biological test without external sources such as an active pump or mechanical valves. A filtration experiment was carried out using artificial blood containing resin particles as an explorer survey. The filtration of microparticles was partially achieved by varying the size of the microgrooves. Although the filtration performance basically depended on the dimensions of the microgrooves, optimization is needed for the microgrooved channel to be applied to actual samples (i.e., whole blood). We believe that our microgroove structure would be useful in a technology involving a paper-based point-of-care diagnostic device for precise reagent manipulation.

\section{Acknowledgements}

This work was supported by the Japan Society for the Promotion of Science KAKENHI (Grant no. 17K14140) and by the Amada foundation.

\section{References}

1. A. W. Martinez, S. T. Phillips, M. J. Butte, and G. M. Whitesides, Angew. Chem. Int. Ed., 2007, 46, 1318.

2. D. M. Cate, W. Dungchai, J. C. Cunningham, J. Volckens, and C. S. Henry, Lab Chip, 2013, 13, 2397.

3. S. Karita and T. Kaneta, Anal. Chem., 2014, 86, 12108.

4. J. Sun, C. Cheng, and Y. Liao, Anal. Sci., 2015, 31, 145.

5. K. Ogawa and T. Kaneta, Anal. Sci., 2016, 32, 31.

6. S. T. Sanjay, M. Dou, J. Sun, and X. Li, Sci. Rep., 2016, 6, 30474. 
(a: $R<w\left(=20.1 \mu \mathrm{m}, F=29.4 \mathrm{~J} \mathrm{~cm}^{-2}\right)$

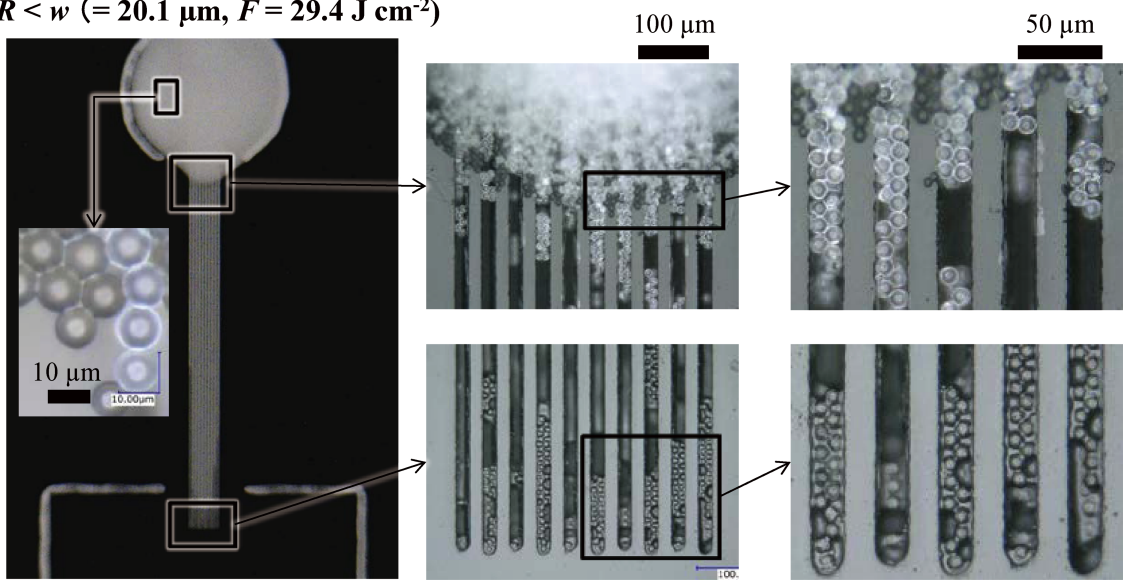

(b: $R>w\left(=9.1 \mu \mathrm{m}, F=9.2 \mathrm{~J} \mathrm{~cm}^{-2}\right)$

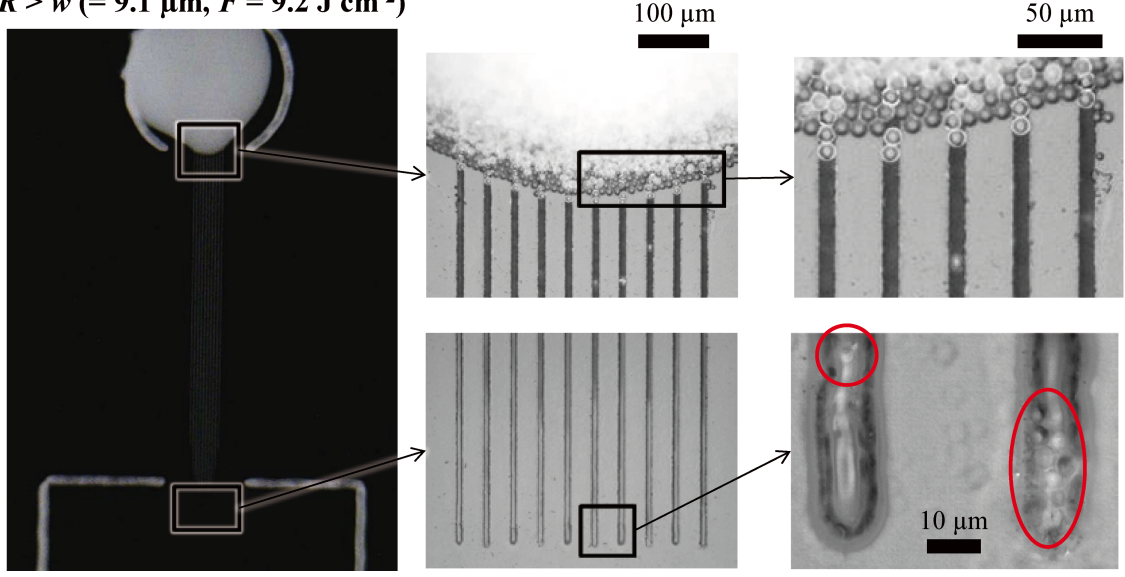

Fig. 7 Micrographs of the microgrooved channel after the injection of artificial blood, where (a) the width $w$ is greater than the particle diameter $R(R \leq w)$ and where (b) the microgrooved channel was prepared such that $R \geq w$.

7. L. H. Mujawar, A. A. Felemban, and M. S. El-Shahawi, Anal. Sci., 2016, 32, 491.

8. W. Chu, Y. Chen, W. Liu, M. Zhao, and H. Li, Sens. Actuators, B, 2017, 250, 324.

9. D. Cheng, X. Zhang, X. Li, L. Hou, and C. Wang, Anal. Sci., 2017, 33, 185.

10. K. Tominaga, S. Arimoto, K. Shimono, T. Yoshioka, F. Mizutani, and T. Yasukawa, Anal. Sci., 2017, 33, 531.

11. X. Y. An, Y. Z. Heng, J. G. Ao, and J. L. Ee, Anal. Sci., 2017, 33, 1.

12. H. Darcy, "Les Fontaines Publiques de la Ville de Dijon", 1856, Dalmont, Paris, 647.

13. H. Noh and S. T. Phillips, Anal. Chem., 2010, 82, 4181.

14. B. R. Lutz, P. Trinh, C. Ball, E. Fu, and P. Yager, Lab Chip, 2011, 11, 4274.

15. E. Fu, T. Liang, P. Spicar-Mihalic, J. Houghtaling, S. Ramachandran, and P. Yager, Anal. Chem., 2012, 84, 4574.

16. I. Jang and S. Song, Lab Chip, 2015, 15, 3405.

17. J. Bico, U. Thiele, and D. Quéré, Colloids Surf., A, 2002, 206, 41.

18. K. Goya, Y. Yamachoshi, Y. Fuchiwaki, M. Tanaka, T. Ooie, K. Abe, and M. Kataoka, Sens. Actuators, B, 2016, 239, 1275.

19. C. Stamm, T. Kachel, N. Pontius, R. Mitzner, T. Quast, K. Holldack, S. Khan, C. Lupulescu, E. F. Aziz, M. Wietstruk, H. A. Dürr, and W. Eberhardt, Nat. Mater, 2007, 6, 740.
20. E. Bulushev, V. Bessmeltsev, A. Dostovalov, N. Goloshevsky, and A. Wolf, Opt. Lasers Eng., 2016, 79, 39.

21. A. Borowiec, H. F. Tiedje, and H. K. Haugen, Appl. Surf. Sci., 2005, 243, 129.

22. G. Li, G. Feng, S. Wang, H. Zhang, Z. Wang, and S. Zhou, Opt.-Int. J. Light Electron Opt., 2017, 140, 953.

23. D. Wu, S. Z. Wu, J. Xu, L. G. Niu, K. Midorikawa, and K. Sugioka, Laser Photon. Rev., 2014, 8, 458.

24. Z. K. Wang, H. Y. Zheng, C. P. Lim, and Y. C. Lam, Appl. Phys. Lett., 2009, 95, 111110.

25. C. De Marco, S. M. Eaton, R. Suriano, S. Turri, M. Levi, R. Ramponi, G. Cerullo, and R. Osellame, ACS Appl. Mater. Interfaces, 2010, 2, 2377.

26. Y. Hanada, T. Ogawa, K. Koike, and K. Sugioka, Lab Chip, 2016, 16, 2481.

27. M. Henyk, N. Vogel, D. Wolfframm, A. Tempel, and J. Reif, Appl. Phys. A Mater. Sci. Process., 1999, 69, 355.

28. J. Reif, F. Costache, M. Henyk, and S. V. Pandelov, Appl. Surf. Sci., 2002, 197-198, 891.

29. D. Dufft, A. Rosenfeld, S. K. Das, R. Grunwald, and J. Bonse, J. Appl. Phys., 2009, 105, 034908.

30. A. Ben-Yakar, A. Harkin, J. Ashmore, R. L. Byer, and H. A. Stone, J. Phys. D. Appl. Phys., 2007, 40, 1447.

31. M. Feinaeugle, A. P. Alloncle, P. Delaporte, C. L. Sones, and R. W. Eason, Appl. Surf. Sci., 2012, 258, 8475. 